\title{
A new weight class and Poincaré inequalities with the Radon measure
}

\author{
Yuming Xing
}

Correspondence: xyuming@hit.edu. $\mathrm{cn}$

Department of Mathematics, Harbin Institute of Technology, Harbin, China

\section{Abstract}

We first introduce and study a new family of weights, the $A(\alpha, \beta, \gamma, E)$-class which contains the well-known $A_{r}(E)$-weight as a proper subset. Then, as applications of the $A(\alpha, \beta, \gamma ; E)$-class, we prove the local and global Poincaré inequalities with the Radon measure for the solutions of the non-homogeneous $A$-harmonic equation which belongs to a kind of the nonlinear partial differential equations.

2000 Mathematics Subject Classification: Primary 26D10; Secondary 35J60; 31B05; 58A10; 46E35.

Keywords: weights, Poincar?é?-type inequality, harmonic equations and differential forms
\end{abstract}

\section{Introduction}

Let $\Omega$ be a domain in $\mathbb{R}^{n}, n \geq 2, B$ be a ball and $\sigma B$ be the ball with the same center and $\operatorname{diam}(\sigma B)=\sigma \operatorname{diam}(B), \sigma>0$. We use $|E|$ to denote the Lebesgue measure of the set $E \subset \mathbb{R}^{n}$. We say $w$ is a weight if $w \in L_{l o c}^{1}\left(\mathbb{R}^{n}\right)$ and $w>0$ a.e. In 1972 , Muckenhoupt [1] introduced the following $A_{r}(E)$-weight in order to study the properties of the Hardy-Littlewood maximal operator. We say a weight $w$ satisfies the $A_{r}(E)$-condition in a subset $E \subset \mathbb{R}^{n}$, where $r>1$, and write $w \in A_{r}(E)$ when

$$
\sup _{B}\left(\frac{1}{|B|} \int_{B} w d x\right)\left(\frac{1}{|B|} \int_{B}\left(\frac{1}{w}\right)^{\frac{1}{r-1}} d x\right)^{r-1}<\infty
$$

where the supremum is over all balls $B \subset E$. Since then, the weight functions have been well studied and widely used in analysis and PDEs, particularly in areas of the measures and integrals, see [2-11]. In 1998, the following $A_{r}(\lambda, E)$-weight class was introduced in [12]. We say that a weight $w$ belongs to the $A_{r}(\lambda, E)$ class, $1<r<\infty$ and $0<\lambda<\infty$, or that $w$ is an $A_{r}(\lambda, E)$-weight, write $w \in A_{r}(\lambda, E)$, if $\sup _{B}\left(\frac{1}{|B|} \int_{B} w^{\lambda} d x\right)\left(\frac{1}{|B|} \int_{B}\left(\frac{1}{w}\right)^{1 /(r-1)} d x\right)^{r-1}<\infty$ for all balls $B \subset E$. Notice that if we choose $\lambda=1$, we find that $A_{r}(1, E)=A_{r}(E)$. In 2000, the following class of $A_{r}^{\lambda}(E)$-weights was introduced in [13]. We say that the weight $w(x)>0$ satisfies the

(c) 2012 Xing; licensee Springer. This is an Open Access article distributed under the terms of the Creative Commons Attribution License (http://creativecommons.org/licenses/by/2.0), which permits unrestricted use, distribution, and reproduction in any medium, provided the original work is properly cited. 
$\sup _{B}\left(\frac{1}{|B|} \int_{B} w d x\right)\left(\frac{1}{|B|} \int_{B} w^{1 /(1-r)} d x\right)^{\lambda(r-1)}<\infty$-condition in $E, r>1$ and $\lambda>0$, and write $w \in A_{r}^{\lambda}(E)$, if $\sup _{B}\left(\frac{1}{|B|} \int_{B} w d x\right)\left(\frac{1}{|B|} \int_{B} w^{1 /(1-r)} d x\right)^{\lambda(r-1)}<\infty$ for any ball $B \subset E$ $\subset \mathbb{R}^{n}$. Also, it is easy to see that $A_{r}^{1}(E)=A_{r}(E)$. Both $A_{r}(\lambda, E)$ and $A_{r}^{\lambda}(E)$ have widely been used in the study of the weighted inequalities and integral estimates, see [4-6,12,13] for example.

\section{The $A(\alpha, \beta, \gamma, E)$-class}

In this section, we first introduce the $A(\alpha, \beta, \gamma, E)$-class which is an extension of the $A_{r}$ $(E)$-weight. Then, we study the properties of this class. We will use the following Hölder inequality repeatedly in this article.

Lemma 2.1. Let $0<\alpha<\infty, 0<\beta<\infty$ and $s^{-1}=\alpha^{-1}+\beta^{-1}$. If $f$ and $g$ are measurable functions on $\mathbb{R}^{n}$, then $\|f g\|_{s, E} \leq\|f\|_{\alpha, E} \cdot\|g\|_{\beta, E}$ for any $E \subset \mathbb{R}^{n}$.

We introduce the following class of functions which is an extension of the several existing classes of weights, such as $A_{r}^{\lambda}(E)$-weights, $A_{r}(\lambda, E)$-weights, and $A_{r}(E)$ weights.

Definition 2.2. We say that a measurable function $g(x)$ defined on a subset $E \subset \mathbb{R}^{n}$ satisfies the $A(\alpha, \beta, \gamma, E)$-condition for some positive constants $\alpha, \beta$, $\gamma$, write $g(x) \in A$ $(\alpha, \beta, \gamma, E)$ if $g(x)>0$ a.e., and

$$
\sup _{B}\left(\frac{1}{|B|} \int_{B} g^{\alpha} d x\right)\left(\frac{1}{|B|} \int_{B} g^{-\beta} d x\right)^{\gamma / \beta}<\infty
$$

where the supremum is over all balls $B \subset E$.

We should notice that there are three parameters in the definition of the $A(\alpha, \beta, \gamma$; $E)$-class. If we choose some special values for these parameters, we may obtain the existing weights. For example, if $\alpha=\lambda, \beta=1 /(r-1)$ and $\gamma=1$ in above definition, the $A(\alpha, \beta, \gamma, E)$-class becomes $A_{r}(\lambda, E)$-weight, that is $A_{r}(\lambda, E)=A(\lambda, 1 /(r-1), 1 ; E)$. Similarly, $A_{r}^{\lambda}(E)=A(1,1 /(r-1), \lambda ; E)$. Also, it is easy to see that the $A(\alpha, \beta, \gamma ; E)$-class reduces to the usual $A_{r}(E)$-weight if $\alpha=\gamma=1$ and $\beta=1 /(r-1)$. Moreover, we have the following theorem which establishes the relationship between the $A_{r}(E)$-weight and the $A(\alpha, \beta, \gamma, E)$-class.

Theorem 2.3. Let $r>1$ be any constant and $E \subset \mathbb{R}^{n}$. Then, (i) There exists a constant $\alpha_{0}>1$ such that $A_{r}(E) \subset A\left(\alpha_{0}, 1 /(r-1), \alpha_{0} ; E\right)$. (ii) For any $\alpha$ with $0<\alpha<1, A_{r}(E)$ $\subset A(\alpha, 1 /(r-1), \alpha ; E)$.

Proof. For $w(x) \in A_{r}(E)$, by the reverse Hölder inequality for the $A_{r}(E)$-weight, there are constants $\alpha_{0}>1$ and $C_{1}>0$ such that

$$
\left(\frac{1}{|B|} \int_{B} w^{\alpha_{0}} d x\right)^{1 / \alpha_{0}} \leq \frac{C_{1}}{|B|} \int_{B} w d x
$$

for all balls $B \subset E$, i.e.,

$$
\frac{1}{|B|} \int_{B} w^{\alpha_{0}} d x \leq C_{2}\left(\frac{1}{|B|} \int_{B} w d x\right)^{\alpha_{0}}
$$


From (2.3) and (1.1), we obtain

$$
\begin{aligned}
& \sup _{B}\left(\frac{1}{|B|} \int_{B} w^{\alpha_{0}} d x\right)\left(\frac{1}{|B|} \int_{B} w^{-\frac{1}{r-1}} d x\right)^{\alpha_{0}(r-1)} \\
& \leq C_{2} \sup _{B}\left(\frac{1}{|B|} \int_{B} w d x\right)^{\alpha_{0}}\left(\frac{1}{|B|} \int_{B} w^{-\frac{1}{r-1}} d x\right)^{\alpha_{0}(r-1)} \\
& \leq C_{2}\left(\sup _{B}\left(\frac{1}{|B|} \int_{B} w d x\right)\left(\frac{1}{|B|} \int_{B}\left(\frac{1}{w}\right)^{\frac{1}{r-1}} d x\right)^{r-1}\right)^{\alpha_{0}}<\infty,
\end{aligned}
$$

where the supremum is over all balls $B \subset E$. Thus, $w \in A\left(\alpha_{0}, 1 /(r-1), \alpha_{0} ; E\right)$. Hence, $A_{r}(E) \subset A\left(\alpha_{0}, 1 /(r-1), \alpha_{0} ; E\right)$. We have completed the proof of the first part of Theorem 2.3. Next, we prove the second part of the theorem. Let $\alpha \in(0,1)$ be any real number. Using the Hölder inequality with $1 / \alpha=1+(1-\alpha) / \alpha$, we have

$$
\left(\int_{B} w^{\alpha} d x\right)^{1 / \alpha} \leq\left(\int_{B} w d x\right)\left(\int_{B} 1 \overline{1-\alpha} d x\right)^{(1-\alpha) / \alpha}
$$

that is

$$
\left(\frac{1}{|B|} \int_{B} w^{\alpha} d x\right)^{1 / \alpha} \leq \frac{1}{|B|} \int_{B} w d x
$$

which can be written as

$$
\frac{1}{|B|} \int_{B} w^{\alpha} d x \leq\left(\frac{1}{|B|} \int_{B} w d x\right)^{\alpha}
$$

Similar to inequality (2.4), using (2.6) and the definitions of the $A_{r}(E)$-weight and the $A(\alpha, \beta, \gamma, E)$-class, we obtain that $A_{r}(E) \subset A(\alpha, 1 /(r-1), \alpha ; E)$ for any $\alpha$ with $0<\alpha<1$. The proof of Theorem 2.3 has been completed.

Example 2.4. Let $\Omega \subset \mathbb{R}^{n}$ be a bounded domain containing the origin and $g(x)=|x|$ $p, x \in \Omega$. We all know that $g(x)=|x|^{p} \in A_{r}(\Omega)$ for some $r>1$ if and only if $-n<p<n$ $(r-1)$. Now, we consider an example in $\mathbb{R}^{2}$, that is $n=2$. Assume that $D \subset \mathbb{R}^{2}$ is a bounded domain containing the origin and $g(x)=|x|^{-3}$ is a function in $D$. Since $p=-3$ $<-2=-n$, then $g(x)=|x|^{-3} \notin A_{r}(D)$ for any $r>1$. However, it is easy to check that $g(x)$ $=|x|^{-3} \in A(\alpha, \beta, \gamma, D)$ for any positive numbers $\alpha, \beta$, $\gamma$ with $0<\alpha<2 / 3$.

Combining Theorem 2.3 and Example 2.4, we find that $A_{r}(E)$ is a proper subset of $A$ $(\alpha, \beta, \gamma, E)$ for any positive constants $\alpha, \beta, \gamma$ and $r$ with $0<\alpha<2 / 3$ and $r>1$.

Theorem 2.5. If $g_{1}(x), g_{2}(x) \in A(\alpha, \beta, \gamma, E)$ for some $\alpha \geq 1, \beta, \gamma>0$ and a subset $E \subset$ $\mathbb{R}^{n}$, then $g_{1}(x)+g_{2}(x) \in A(\alpha, \beta, \gamma, E)$.

Proof. Let $g_{1}(x), g_{2}(x) \in A(\alpha, \beta, \gamma ; E)$. By Minkowski inequality, we find that

$$
\left(\int_{B}\left|g_{1}+g_{2}\right|^{\alpha} d x\right)^{\frac{1}{\alpha}} \leq\left(\int_{B}\left|g_{1}\right|^{\alpha} d x\right)^{\frac{1}{\alpha}}+\left(\int_{B}\left|g_{2}\right|^{\alpha} d x\right)^{\frac{1}{\alpha}} .
$$


King Journal of Inequalities and Applications 2012, 2012:32

Page 4 of 11

http://www.journalofinequalitiesandapplications.com/content/2012/1/32

Since $|a+b|^{s} \leq 2^{s}\left(|a|^{s}+|b|^{s}\right)$ for any constants $a, b$, s with $s>0$, from (2.7), we have

$$
\begin{aligned}
\int_{B}\left(g_{1}+g_{2}\right)^{\alpha} d x & \leq\left(\left(\int_{B}\left|g_{1}\right|^{\alpha} d x\right)^{\frac{1}{\alpha}}+\left(\int_{B}\left|g_{2}\right|^{\alpha} d x\right)^{\frac{1}{\alpha}}\right)^{\alpha} \\
& \leq 2^{\alpha}\left(\int_{B}\left|g_{1}\right|^{\alpha} d x+\int_{B}\left|g_{2}\right|^{\alpha} d x\right) .
\end{aligned}
$$

Note that $g_{1}(x), g_{2}(x) \in A(\alpha, \beta, \gamma, E)$. Using (2.8), we obtain

$$
\begin{aligned}
& \sup _{B}\left(\frac{1}{|B|} \int_{B}\left(g_{1}+g_{2}\right)^{\alpha} d x\right)\left(\frac{1}{|B|} \int_{B}\left(g_{1}+g_{2}\right)^{-\beta} d x\right)^{\gamma / \beta} \\
& \leq \sup _{B} 2^{\alpha}\left(\frac{1}{|B|} \int_{B}\left|g_{1}\right|^{\alpha} d x+\frac{1}{|B|} \int_{B}\left|g_{2}\right|^{\alpha} d x\right)\left(\frac{1}{|B|} \int_{B}\left(g_{1}+g_{2}\right)^{-\beta} d x\right)^{\gamma / \beta} \\
& \leq \sup _{B} 2^{\alpha}\left(\frac{1}{|B|} \int_{B} g_{1}^{\alpha} d x\left(\frac{1}{|B|} \int_{B} g_{1}^{-\beta} d x\right)^{\gamma / \beta}+\frac{1}{|B|} \int_{B} g_{2}^{\alpha} d x\left(\frac{1}{|B|} \int_{B} g_{2}^{-\beta} d x\right)^{\gamma / \beta}\right) \\
& <\infty .
\end{aligned}
$$

Thus, $g_{1}(x)+g_{2}(x) \in A(\alpha, \beta, \gamma, E)$. The proof of Theorem 2.5 has been completed. Theorem 2.6. Let $g_{1}(x) \in A\left(\alpha_{1}, \beta_{1}, \alpha_{1} \gamma, E\right)$ and $g_{2}(x) \in A\left(\alpha_{2}, \beta_{2}, \alpha_{2} \gamma, E\right)$ for some $\gamma>$ 0 and any subset $E \subset \mathbb{R}^{n}$, where $\alpha_{i}, \beta_{i}>0, i=1,2$, and $\frac{1}{\alpha}=\frac{1}{\alpha_{1}}+\frac{1}{\alpha_{2}}, \frac{1}{\beta}=\frac{1}{\beta_{1}}+\frac{1}{\beta_{2}}$. Then, $g_{1}(x) g_{2}(x) \in A(\alpha, \beta, \alpha \gamma, E)$.

Proof. Using Lemma 2.1 with $\frac{1}{\alpha}=\frac{1}{\alpha_{1}}+\frac{1}{\alpha_{2}}$ and $\frac{1}{\beta}=\frac{1}{\beta_{1}}+\frac{1}{\beta_{2}}$, respectively, we have

$$
\begin{aligned}
& \left(\int_{B}\left(g_{1} g_{2}\right)^{\alpha} d x\right)^{1 / \alpha} \leq\left(\int_{B} g_{1}^{\alpha_{1}} d x\right)^{1 / \alpha_{1}}\left(\int_{B} g_{2}^{\alpha_{2}} d x\right)^{1 / \alpha_{2}}, \\
& \left(\int_{B}\left(g_{1} g_{2}\right)^{-\beta} d x\right)^{\gamma / \beta} \leq\left(\int_{B} g_{1}^{-\beta} d x\right)^{\gamma / \beta_{1}}\left(\int_{B} g_{2}^{-\beta_{2}} d x\right)^{\gamma / \beta_{2}} .
\end{aligned}
$$

Combining (2.9) and (2.10) yields

$$
\begin{aligned}
& \left(\int_{B}\left(g_{1} g_{2}\right)^{\alpha} d x\right)^{1 / \alpha}\left(\int_{B}\left(g_{1} g_{2}\right)^{-\beta} d x\right)^{\gamma / \beta} \\
& \leq\left(\int_{B} g_{1}^{\alpha_{1}} d x\right)^{1 / \alpha_{1}}\left(\int_{B} g_{1}^{-\beta_{1}} d x\right)^{\gamma / \beta_{1}}\left(\int_{B} g_{2}^{\alpha_{2}} d x\right)^{1 / \alpha_{2}}\left(\int_{B} g_{2}^{-\beta_{2}} d x\right)^{\gamma / \beta_{2}}
\end{aligned}
$$

which is equivalent to

$$
\begin{aligned}
& \left(\int_{B}\left(g_{1} g_{2}\right)^{\alpha} d x\left(\int_{B}\left(g_{1} g_{2}\right)^{-\beta} d x\right)^{\alpha \gamma / \beta}\right)^{1 / \alpha} \\
& \leq\left(\int_{B} g_{1}^{\alpha_{1}} d x\left(\int_{B} g_{1}^{-\beta_{1}} d x\right)^{\alpha_{1} \gamma / \beta_{1}}\right)^{1 / \alpha_{1}}\left(\int_{B} g_{2}^{\alpha_{2}} d x\left(\int_{B} g_{2}^{-\beta_{2}} d x\right)^{\alpha_{2} \gamma / \beta_{2}}\right)^{1 / \alpha_{2}} .
\end{aligned}
$$


Noticing that $g_{1}(x) \in A\left(\alpha_{1}, \beta_{1}, \alpha_{1} \gamma, E\right)$ and $g_{2}(x) \in A\left(\alpha_{2}, \beta_{2}, \alpha_{2} \gamma, E\right)$, we obtain

$$
\begin{aligned}
& \sup _{B}\left(\frac{1}{|B|} \int_{B}\left(g_{1} g_{2}\right)^{\alpha} d x\right)\left(\frac{1}{|B|} \int_{B}\left(g_{1} g_{2}\right)^{-\beta} d x\right)^{\alpha \gamma / \beta} \\
& \left.\leq \sup _{B}\left(\frac{1}{|B|} \int_{B} g_{1}^{\alpha_{1}} d x\right)\left(\frac{1}{|B|} \int_{B} g_{1}^{-\beta_{1}} d x\right)^{\frac{\alpha_{1} \gamma}{\beta_{1}}}\right)^{\frac{\alpha}{\alpha_{1}}}\left(\sup _{B}\left(\frac{1}{|B|} \int_{B} g_{2}^{\alpha_{2}} d x\right)\left(\frac{1}{|B|} \int_{B} g_{2}^{-\beta_{2}} d x\right)^{\frac{\alpha_{2} \gamma}{\beta_{2}}}\right)^{\frac{\alpha}{\alpha_{2}}}
\end{aligned}
$$

Thus, $g_{1}(x) g_{2}(x) \in A(\alpha, \beta, \alpha \gamma, E)$. The proof of Theorem 2.6 has been completed.

Proposition 2.7. Let $0<p<1$ and $g(x) \in A(\alpha, \beta p, \gamma, E)$. Then, $g^{p}(x) \in A(\alpha, \beta, \gamma, E)$.

Proof. Using Lemma 2.1 with $\frac{1}{\alpha p}=\frac{1}{\alpha}+\frac{1-p}{\alpha p}$ yields

$$
\left(\int_{B} g^{\alpha p} d x\right)^{1 / \alpha p} \leq|B|^{(1-p) / \alpha p}\left(\int_{B} g^{\alpha} d x\right)^{1 / \alpha}
$$

that is

$$
\frac{1}{|B|} \int_{B}\left(g^{p}\right)^{\alpha} d x \leq\left(\frac{1}{|B|} \int_{B} g^{\alpha} d x\right)^{p} .
$$

Since $g(x) \in A(\alpha, \beta p, \gamma, E)$, using (2.14), we find that

$$
\begin{aligned}
& \sup _{B}\left(\frac{1}{|B|} \int_{B}\left(g^{p}\right)^{\alpha} d x\right)\left(\frac{1}{|B|} \int_{B}\left(g^{p}\right)^{-\beta} d x\right)^{\gamma / \beta} \\
& \leq \sup _{B}\left(\frac{1}{|B|} \int_{B} g^{\alpha} d x\right)^{p}\left(\frac{1}{|B|} \int_{B} g^{-\beta p} d x\right)^{\gamma / \beta} \\
& \leq \sup _{B}\left(\left(\frac{1}{|B|} \int_{B} g^{\alpha} d x\right)\left(\frac{1}{|B|} \int_{B} g^{-\beta p} d x\right)^{\gamma / \beta p}\right)^{p} \\
& \leq\left(\sup _{B}\left(\frac{1}{|B|} \int_{B} g^{\alpha} d x\right)\left(\frac{1}{|B|} \int_{B} g^{-\beta p} d x\right)^{\gamma / \beta p}\right)^{p} \\
& <\infty .
\end{aligned}
$$

Therefore, $g^{p}(x) \in A(\alpha, \beta, \gamma, E)$. The proof of Proposition 2.7 has been completed.

Let $\alpha, \beta, \gamma>0$ be any constants. It is easy to prove that (i) $\frac{1}{g(x)} \in A(\alpha, \beta, \gamma ; E)$ if and only if $g(x) \in A(\beta, \alpha, \alpha \beta / \gamma, E)$. (ii) $g^{p}(x) \in A(\alpha, \beta, \gamma, E)$ if and only if $g(x) \in A(\alpha p, \beta p$, $\gamma p$; E) for any constant $p>0$. Also, using the Hölder inequality and the definition of the $A(\alpha, \beta, \gamma, E)$-class, we can prove the following monotone properties of the $A(\alpha, \beta$, $\gamma, E)$-class.

Proposition 2.8. If $\alpha_{1}<\alpha_{2}$, then $A\left(\alpha_{2}, \beta, \gamma, E\right) \subset A\left(\alpha_{1}, \beta, \gamma, E\right)$ for any $\beta, \gamma>0$. If $\beta_{1}$ $<\beta_{2}$, then $A\left(\alpha, \beta_{2}, \gamma, E\right) \subset A\left(\alpha, \beta_{1}, \gamma, E\right)$ for any $\alpha, \gamma>0$.

From Theorem 2.3 and Proposition 2.8, we know that for every $r>1$, there exists a constant $\alpha_{0}>1$ such that $A_{r}(E) \subset A(\alpha, 1 /(r-1), \alpha$; $E)$ for any $\alpha$ with $0<\alpha<\alpha_{0}$.

\section{Local Poincaré inequalities}

As applications of the $A(\alpha, \beta, \gamma, E)$-class, we prove the local Poincaré inequalities with the Radon measure for the differential forms satisfying the non-homogeneous $A$-harmonic equation. Differential forms are extensions of functions in $\mathbb{R}^{n}$. For example, the 
function $u\left(x_{1}, x_{2}, \ldots, x_{n}\right)$ is called a 0 -form. The 1 -form $u(x)$ in $\mathbb{R}^{n}$ can be written as $u(x)=\sum_{i=1}^{n} u_{i}\left(x_{1}, x_{2}, \ldots, x_{n}\right) d x_{i}$. If the coefficient functions $u_{i}\left(x_{1}, x_{2}, \ldots, x_{n}\right), i=1,2, \ldots, n$, are differentiable, then $u(x)$ is called a differential 1 -form. Similarly, a differential $k$ form $u(x)$ is generated by $\left\{d x_{i_{1}} \wedge d x_{i_{2}} \wedge \cdots \wedge d x_{i_{k}}\right\}, k=1,2, \ldots, n$, that is, $u(x)=\sum_{I} u_{I}(x) d x_{I}=\sum u_{i_{1} i_{2} \ldots i_{k}}(x) d x_{i_{1}} \wedge d x_{i_{2}} \wedge \cdots \wedge d x_{i^{\prime}}$, where $I=\left(i_{1}, i_{2}, \ldots, i_{k}\right), 1 \leq i_{1}$ $<i_{2}<\ldots<i_{k} \leq n$. Let $\Lambda^{l}=\Lambda^{l}\left(\mathbb{R}^{n}\right)$ be the set of all $l$-forms in $\mathbb{R}^{n}$ and $L^{p}\left(\Omega, \Lambda^{l}\right)$ be the $l$ forms $u(x)=\Sigma_{I} u_{I}(x) d x_{I}$ in $\Omega$ satisfying $\int_{\Omega}\left|u_{I}\right|^{p}<\infty$ for all ordered $l$-tuples $I, l=$ $1,2, \ldots, n$. We denote the exterior derivative by $d$ and the Hodge star operator by *. The Hodge codifferential operator $d^{*}$ is given by $d^{*}=(-1)^{n l+1} * d^{*}, l=0,1, \ldots, n-1$. We consider here the solutions to the nonlinear partial differential equation

$$
d^{*} A(x, d u)=B(x, d u)
$$

which is called non-homogeneous $A$-harmonic equation, where $A: \Omega \times \wedge^{l}\left(\mathbb{R}^{n}\right) \rightarrow \Lambda^{l}$ $\left(\mathbb{R}^{n}\right)$ and $B: \Omega \times \Lambda^{l}\left(\mathbb{R}^{n}\right) \rightarrow \Lambda^{l-1}\left(\mathbb{R}^{n}\right)$ satisfy the conditions: $|A(x, \xi)| \leq a|\xi|^{p-1}, A(x, \xi) \cdot \xi$ $\geq|\xi|^{p}$ and $|B(x, \xi)| \leq b|\xi|^{p-1}$ for almost every $x \in \Omega$ and all $\xi \in \Lambda^{l}\left(\mathbb{R}^{n}\right)$. Here $a, b>0$ are constants and $1<p<\infty$ is a fixed exponent associated with (3.1). A solution to (3.1) is an element of the Sobolev space $W_{l o c}^{1, p}\left(\Omega, \wedge^{l-1}\right)$ such that $\int_{\Omega} A(x, d u) \cdot d \phi+B$ $(x, d u) \cdot \phi=0$ for all $\varphi \in W_{l o c}^{1, p}\left(\Omega, \wedge^{l-1}\right)$ with compact support. If $u$ is a function (0form) in $\mathbb{R}^{n}$, the equation (3.1) reduces to

$$
\operatorname{div} A(x, \nabla u)=B(x, \nabla u) .
$$

If the operator $B=0$, Equation (3.1) becomes $d^{*} A(x, d u)=0$, which is called the (homogeneous) $A$-harmonic equation. Let $A: \Omega \times \Lambda^{l}\left(\mathbb{R}^{n}\right) \rightarrow \Lambda^{l}\left(\mathbb{R}^{n}\right)$ be defined by $A(x$, $\xi)=\xi|\xi|^{p-2}$ with $p>1$. Then, $A$ satisfies the required conditions and $d^{*} A(x, d u)=0$ becomes the $p$-harmonic equation $d^{*}\left(d u|d u|^{p-2}\right)=0$ for differential forms. See [5,6,9-16] for recent results on the solutions to the different versions of the $A$-harmonic equation. The operator $K_{y}$ with the case $y=0$ was first introduced by Cartan [17]. Then, it was extended to the following version in [18]. Let $D$ be a convex and bounded domain. To each $y \in D$ there corresponds a linear operator $K_{y}: C^{\infty}\left(D, \Lambda^{l}\right) \rightarrow C^{\infty}\left(D, \wedge^{l-}\right.$ $\left.{ }^{1}\right)$ defined by $\left(K_{y} u\right)\left(x ; \xi_{1}, \ldots, \xi_{l-1}\right)=\int_{0}^{1} t^{l-1} u\left(t x+y-t y ; x-y, \xi_{1}, \ldots, \xi_{l-1}\right) d t$. A homotopy operator $T: C^{\infty}\left(D, \wedge^{l}\right) \rightarrow C^{\infty}\left(D, \wedge^{l-1}\right)$ is defined by averaging $K_{y}$ over all points $y \in D$ : $T u=\int_{D} \phi(y) K_{y} u d y$, where $\phi \in C_{0}^{\infty}(D)$ is normalized so that $\int_{D} \phi(y) d y=1$. The $l$-form is defined by $\omega_{D}=|D|^{-1} \int_{D} \omega(y) d y, l=0$, and $\omega_{D}=d(T \omega), l=1,2, \ldots, n$ for all $\omega \in L^{p}$ $\left(D, \wedge^{l}\right), 1 \leq p \leq \infty$. For any differential form $u \in L_{l o c}^{s}\left(D, \wedge^{l}\right), l=1,2, \ldots, n, 1<s<\infty$, we have

$$
\|T u\|_{s, D} \leq C|D| \operatorname{diam}(D)\|u\|_{s, D} .
$$

Lemma 3.1. [14] Let $u$ be a differential form satisfying the non-homogeneous A-harmonic equation (3.1) in $\Omega, \sigma>1$ and $0<s, t<\infty$. Then, there exists a constant $C$, independent of $u$, such that $\|d u\|_{s, B} \leq C|B|^{(t-s) / s t}|| d u \|_{t, \sigma B}$ for all balls or cubes $B$ with $\sigma B$ $\subset \Omega$.

Theorem 3.2. Let $u \in L_{l o c}^{s}\left(\Omega, \wedge^{l}\right)$ be a solution of the non-homogeneous A-harmonic equation (3.1) in a domain $\Omega, d u \in L_{\text {loc }}^{s}\left(\Omega, \wedge^{l+1}\right), l=0,1, \ldots, n-1$ and $1<s<\infty$. Then, there exists a constant $C$, independent of $u$, such that 


$$
\left(\int_{B}\left|u-u_{B}\right|^{s} d \mu\right)^{1 / s} \leq C|B| \operatorname{diam}(B)\left(\int_{\sigma B}|d u|^{s} d \mu\right)^{1 / s}
$$

for all balls $B$ with $\sigma B \subset \Omega$, where the Radon measure $\mu$ is defined by $d \mu=g(x) d x$ and $g \in A(\alpha, \beta, \alpha ; \Omega), \alpha>1, \beta>0$.

Proof. By the decomposition theorem of differential forms, we have $u=d(T u)+T$ $(d u)=u_{B}+T(d u)$, where $d$ is the exterior differential operator and $T$ is the homotopy operator.

From (3.3), we obtain

$$
\left\|u-u_{B}\right\|_{t, B}=\|T(d u)\|_{t, B} \leq C_{1}|B| \operatorname{diam}(B)\|d u\|_{t, B}
$$

for any $t>1$. Now, choose $t=\alpha s /(\alpha-1)$, then, $t>s$. Using the Hölder inequality and (3.5), we obtain

$$
\begin{aligned}
\left(\int_{B}\left|u-u_{B}\right|^{s} d \mu\right)^{1 / s} & =\left(\int_{B}\left|u-u_{B}\right|^{s} g(x) d x\right)^{1 / s} \\
& =\left(\int_{B}\left(\left|u-u_{B}\right| g^{1 / s}(x)\right)^{s} d x\right)^{1 / s} \\
& \leq\left(\int_{B}\left|u-u_{B}\right|^{t} d x\right)^{1 / t}\left(\int_{B} g^{t /(t-s)}(x) d x\right)^{(t-s) / s t} \\
& \leq C_{2}|B| \operatorname{diam}(B)\|d u\|_{t, B}\left(\int_{B} g^{\alpha}(x) d x\right)^{1 / \alpha s} .
\end{aligned}
$$

Let $m=\beta s /(1+\beta)$, then $0<m<s$. From Lemma 3.1, we have

$$
\|d u\|_{t, B} \leq C_{3}|B| \frac{m-t}{m t}\|d u\| m, \sigma_{1} B,
$$

where $\sigma_{1}>1$ is a constant. Using the Hölder inequality again, we find that

$$
\begin{aligned}
\|d u\|_{m, \sigma_{1} B} & =\left(\int_{\sigma_{1} B}\left(|d u|(g(x))^{1 / s}(g(x))^{-1 / s}\right)^{m} d x\right)^{1 / m} \\
& \leq\left(\int_{\sigma_{1} B}|d u|^{s} g(x) d x\right)^{1 / s}\left(\int_{\sigma_{1} B}\left(g^{-1 / s}(x)\right)^{\frac{m s}{s-m}} d x\right)^{\frac{s-m}{m s}} \\
& \leq\left(\int_{\sigma_{1} B}|d u|^{s} g(x) d x\right)^{1 / s}\left(\int_{\sigma_{1} B}(g(x))^{\frac{-m}{s-m}} d x\right)^{\frac{s-m}{m s}} \\
& \leq\left(\int_{\sigma_{1} B}|d u|^{s} d \mu\right)^{1 / s}\left(\int_{\sigma_{1} B} g^{-\beta}(x) d x\right)^{1 / \beta s} .
\end{aligned}
$$


Since $g \in A(\alpha, \beta, \alpha ; \Omega)$, it follows that

$$
\begin{aligned}
& \left(\int_{B} g^{\alpha}(x) d x\right)^{1 / \alpha s}\left(\int_{\sigma_{1} B} g^{-\beta}(x) d x\right)^{1 / \beta s} \\
& \leq\left(\left(\int_{\sigma_{1} B} g^{\alpha}(x) d x\right)\left(\int_{\sigma_{1} B} g^{-\beta}(x) d x\right)^{\alpha / \beta}\right)^{1 / \alpha s} \\
& =\left(\left|\sigma_{1} B\right|^{1+\frac{\alpha}{\beta}}\left(\frac{1}{\left|\sigma_{1} B\right|} \int_{\sigma_{1} B} g^{\alpha}(x) d x\right)\left(\frac{1}{\left|\sigma_{1} B\right|} \int_{\sigma_{1} B} g^{-\beta}(x) d x\right)^{\alpha / \beta}\right)^{1 / \alpha s} \\
& \leq C_{4}|B|^{1 / \alpha s+1 / \beta s} .
\end{aligned}
$$

Combining (3.6), (3.7), and (3.8) and using (3.9), we have

$$
\begin{aligned}
& \left(\int_{B}|u-u B|^{s} d \mu\right)^{1 / s} \\
& \leq C_{5}|B| \operatorname{diam}(B)|B| \frac{m-t}{m t}\left(\int_{\sigma_{1} B}|d u|^{s} d \mu\right)^{1 / s}\left(\int_{B} g^{\alpha}(x) d x\right)^{1 / \alpha s}\left(\int_{\sigma_{1} B} g^{-\beta}(x) d x\right)^{1 / \beta s} \\
& \leq C_{5} \operatorname{diam}(B)|B|{ }^{1+\frac{1}{t}}-\frac{1}{m}\left(\int_{\sigma_{1} B}|d u|^{s} d \mu\right)^{1 / s}\left(\left(\int_{B} g^{\alpha}(x) d x\right)\left(\int_{\sigma_{1} B} g^{-\beta}(x) d x\right)^{\alpha / \beta}\right)^{1 / \alpha s} \\
& \leq C_{6}|B| \operatorname{diam}(B)\left(\int_{\sigma_{1} B}|d u|^{s} d \mu\right)^{1 / s},
\end{aligned}
$$

that is

$$
\left(\int_{B}\left|u-u_{B}\right|^{s} d \mu\right)^{1 / s} \leq C_{6}|B| \operatorname{diam}(B)\left(\int_{\sigma_{1} B}(d u)^{s} d \mu\right)^{1 / s} .
$$

We have completed the proof of Theorem 3.2.

Let $g(x)=\frac{1}{\left|x-x_{B}\right|^{\lambda}}$, where $x_{B}$ be the center of the ball $B \subset \Omega$ and $0<\lambda<\frac{n}{\alpha}, \alpha>1$.

Then, $g(x) \in A(\alpha, \beta, \alpha ; \Omega)$. From Theorem 3.2, we have the following corollary.

Corollary 3.3. Let $u \in L_{l o c}^{s}\left(\Omega, \wedge^{l}\right)$ be a solution of the non-homogeneous A-harmonic equation (3.1) in a domain $\Omega, d u \in L_{l o c}^{s}\left(\Omega, \wedge^{l+1}\right), l=0,1, \ldots, n-1$ and $1<s<\infty$. Then, there exists a constant $C$, independent of $u$, such that

$$
\left(\int_{B}\left|u-u_{B}\right|^{s} d \mu\right)^{1 / s} \leq C|B| \operatorname{diam}(B)\left(\int_{\sigma B}|d u|^{s} d \mu\right)^{1 / s}
$$

for all balls $B$ with $\sigma B \subset \Omega$, where the Radon measure $\mu$ is defined by $d \mu=\frac{1}{\left|x-x_{B}\right|^{\lambda}} d x, x_{B}$ is the center of the ball $B \subset \Omega, 0<\lambda<\frac{n}{\alpha}$ and $\alpha>1$ is a constant.

\section{Global Poincaré inequalities}

In this section, we will prove the global Poincaré inequalities with the Radon measure for solutions of the nonhomogeneous $A$-harmonic equation in $L^{s}(\mu)$-averaging domains. In 1989, Staples [19] introduced the following $L^{S}$-averaging domains.

Definition 4.1. A proper subdomain $\Omega \subset \mathbb{R}^{n}$ is called an $L^{s}$-averaging domain, $s \geq 1$, if there exists a constant $\mathrm{C}$ such that 


$$
\left(\frac{1}{|\Omega|} \int_{\Omega}\left|u-u_{\Omega}\right|^{s} d x\right)^{1 / s} \leq C \sup _{B \subset \Omega}\left(\frac{1}{|B|} \int_{B}\left|u-u_{B}\right|^{s} d x\right)^{1 / s}
$$

for all $u \in L_{l o c}^{s}(\Omega)$.

Also, in [19], the $L^{s}$-averaging domain is characterized in terms of the quasi-hyperbolic metric. Particularly, Staples proved that any John domain is $L^{s}$-averaging domain, see [20] for more results on the averaging domains. In [15], the $L^{s}$-averaging domains were extended to the following $L^{s}(\mu)$-averaging domains.

Definition 4.2. We call a proper subdomain $\Omega \subset \mathbb{R}^{n}$ an $L^{s}(\mu)$-averaging domain, $s \geq$ 1 , if there exists a constant $C$ such that

$$
\left(\frac{1}{\mu(\Omega)} \int_{\Omega}\left|u-u_{B_{0}}\right|^{s} d \mu\right)^{1 / s} \leq C \sup _{B \subset \Omega}\left(\frac{1}{\mu(B)} \int_{B}\left|u-u_{B}\right|^{s} d x\right)^{1 / s}
$$

for some ball $B_{0} \subset \Omega$ and all $u \in L_{l o c}^{s}(\Omega ; \mu)$, where the Radon measure $\mu(x)$ is defined by $d \mu=w(x) d x$ and $w(x)$ is a weight. Here, the supremum is over all balls $B$ with $B \subset$ $\Omega$.

Theorem 4.3. Let $u \in L^{S}\left(\Omega, \Lambda^{0}\right)$ be a solution of the non-homogeneous $A$-harmonic equation (3.2) in a domain $\Omega, d u \in L^{s}\left(\Omega, \Lambda^{1}\right), 1<s<\infty$. Then, there exists a constant $C$, independent of $u$, such that

$$
\left(\int_{\Omega}\left|u-u_{B_{0}}\right|^{s} d \mu\right)^{1 / s} \leq C(\mu(\Omega))^{1+1 / n}\left(\int_{\Omega}|d u|^{s} d \mu\right)^{1 / s}
$$

for any $L^{s}(\mu)$-averaging domain $\Omega \subset \mathbb{R}^{n}$ with $\mu(\Omega)<\infty$, where $B_{0}$ is some ball appearing in Definition 4.2 and the Radon measure $\mu$ is defined by $d \mu=g(x) d x, g(x) \in$ $A(\alpha, \beta, \alpha ; \Omega), \alpha>1, \beta>0$.

Proof. We may assume $g(x) \geq 1$ a.e. in $\Omega$. Otherwise, let $\Omega_{1}=\Omega \cap\{x \in \Omega: 0<g(x)$ $<1\}$ and $\Omega_{2}=\Omega \cap\{x \in \Omega: g(x) \geq 1\}$. Then, $\Omega=\Omega_{1} \cup \Omega_{2}$. We define $G(x)$ by

$$
G(x)= \begin{cases}1, & x \in \Omega_{1} \\ g(x), & x \in \Omega_{2}\end{cases}
$$

Then, $G(x) \geq g(x)$ and it is easy to check that $g(x) \in A(\alpha, \beta, \alpha ; \Omega)$ if and only if $G(x)$ $\in A(\alpha, \beta, \alpha ; \Omega)$.

Thus,

$$
\begin{aligned}
\left(\int_{\Omega}\left|u-u_{B_{0}}\right|^{s} d \mu\right)^{1 / s} & =\left(\int_{\Omega}\left|u-u_{B_{0}}\right|^{s} g(x) d x\right)^{1 / s} \\
& \leq\left(\int_{\Omega}\left|u-u_{B_{0}}\right|^{s} G(x) d x\right)^{1 / s}
\end{aligned}
$$

with $G(x) \geq 1$. Hence, we may suppose that $g(x) \geq 1$ a.e. in $\Omega$. Thus, for any $D \subset \Omega$, we have

$$
\mu(D)=\int_{D} d \mu=\int_{D} g(x) d x \geq \int_{D} d x=|D| .
$$


Note that $\operatorname{diam}(B)=C_{1}|B|^{1 / n}$. From Theorem 3.2, we obtain

$$
\left(\frac{1}{|B|} \int_{B}\left|u-u_{B}\right|^{s} d \mu\right)^{1 / s} \leq C_{2}|B|^{1+1 / n-1 / s}\left(\int_{\sigma B}|d u|^{s} d \mu\right)^{1 / s}
$$

By definition of the $L^{s}(\mu)$-averaging domain, (4.3), (4.4) and noticing that $1+1 / n-$ $1 / s>0$, we find that

$$
\begin{aligned}
\left(\frac{1}{\mu(\Omega)} \int_{\Omega}\left|u-u_{B_{0}}\right|^{s} d \mu\right)^{1 / s} & \leq C_{3} \sup _{B \subset \Omega}\left(\frac{1}{\mu(B)} \int_{B}\left|u-u_{B}\right|^{s} d \mu\right)^{1 / s} \\
& \leq C_{3} \sup _{B \subset \Omega}\left(\frac{1}{|B|} \int_{B}\left|u-u_{B}\right|^{s} d \mu\right)^{1 / s} \\
& \leq C_{4} \sup _{B \subset \Omega}|B|^{1+1 / n-1 / s}\left(\int_{\sigma B}|d u|^{s} d \mu\right)^{1 / s} \\
& \leq C_{4}|\Omega|^{1+1 / n-1 / s} \sup \left(\int_{B \subset \Omega}|d u|^{s} d \mu\right)^{1 / s} \\
& \leq C_{4}|\Omega|^{1+1 / n-1 / s}\left(\int_{\Omega}|d u|^{s} d \mu\right)^{1 / s} \\
& \leq C_{4}(\mu(\Omega))^{1+1 / n-1 / s}\left(\int_{\Omega}|d u|^{s} d \mu\right)^{1 / s}
\end{aligned}
$$

that is

$$
\left(\int_{\Omega}\left|u-u_{B_{0}}\right|^{s} d \mu\right)^{1 / s} \leq C(\mu(\Omega))^{1+1 / n}\left(\int_{\Omega}|d u|^{s} d \mu\right)^{1 / s} .
$$

The proof of Theorem 4.3 has been completed.

In [15], it has been proved that any John domain is an $L^{s}(\mu)$-averaging domain. Hence, we have the following corollary.

Corollary 4.4. Let $u \in L^{s}\left(\Omega, \Lambda^{0}\right)$ be a solution of the non-homogeneous A-harmonic equation (3.2) in a John domain $\Omega$ with $\mu(\Omega)<\infty$, du $\in L^{s}\left(\Omega, \wedge^{1}\right), 1<s<\infty$. Then, there exists a constant $C$, independent of $u$, such that

$$
\left(\int_{\Omega}\left|u-u_{B_{0}}\right|^{s} d \mu\right)^{1 / s} \leq C\left(\int_{\Omega}|d u|^{s} d \mu\right)^{1 / s},
$$

where $B_{0}$ is some ball appearing in Definition 4.2 and the Radon measure $\mu$ is defined by $d \mu=g(x) d x$ and $g(x) \in A(\alpha, \beta, \alpha ; \Omega), \alpha>1, \beta>0$.

Example 4.5. Since the usual $p$-harmonic equation $\operatorname{div}\left(\nabla u|\nabla u|^{p-2}\right)=0$ and the $A$ harmonic equation $\operatorname{div} A(x, \nabla u)=0$ for functions are the special cases of the nonhomogeneous $A$-harmonic equation, all results proved in Sections 3 and 4 are still true for $p$-harmonic functions and $A$-harmonic functions.

Remark. (i) Since an $L^{S}$-averaging domain is a special $L^{S}(\mu)$-averaging domain, then the inequality (4.1) still holds in any $L^{s}$-averaging domain. (ii) Since $\mu(\Omega)<\infty$, the inequality (4.1) can be written as

$$
\left(\int_{\Omega}\left|u-u_{B_{0}}\right|^{s} d \mu\right)^{1 / s} \leq C\left(\int_{\Omega}|d u|^{s} d \mu\right)^{1 / s}
$$


where $\Omega$ is an $L^{s}(\mu)$-averaging domain $\Omega \subset \mathbb{R}^{n}$ with $\mu(\Omega)<\infty$ and $B_{0}$ is some ball appearing in Definition 4.2, and the Radon measure $\mu$ is defined by $d \mu=g(x) d x$ and $g$ $(x) \in A(\alpha, \beta, \alpha ; \Omega), \alpha>1, \beta>0$. (iii) The inequalities obtained in this article are extensions of the usual $A_{r}(E)$-weighted inequalities since the $A_{r}(E)$ is a proper subset of the $A(\alpha, \beta, \alpha ; E)$-class which can be used to extend many results with the $A_{r}(E)$-weight into the $A(\alpha, \beta, \alpha ; E)$-weight.

\section{Acknowledgements}

I would like to thank Professor Shusen Ding for his precious and thoughtful suggestions which greatly improved the presentation of the article. This work was supported by the Foundation of Education Department of Heilongjiang Province in 2011 (\#12511111).

\section{Competing interests}

The author declares that he has no competing interests.

Received: 11 May 2011 Accepted: 15 February 2012 Published: 15 February 2012

\section{References}

1. Muckenhoupt, B: Weighted norm inequalities for the Hardy maximal function. Trans Am Math Soc. 165, $207-226$ (1972)

2. Agarwal, RP, O'Regan, D, Shakhmurov, V: Separable anisotropic differential operators in weighted abstract spaces and applications. J Math Anal Appl. 338, 970-983 (2008). doi:10.1016/j.jmaa.2007.05.078

3. Agarwal, RP, Diagana, T, Hernandez, EM: Weighted pseudo almost periodic solutions to some partial neutral functional differential equations. J Nonlinear Convex Anal. 8, 397-415 (2007)

4. Bao, G: $A_{r}(\lambda)$-weighted integral inequalities for A-harmonic tensors. J Math Anal Appl. 247, 466-477 (2000). doi:10.1006/ jmaa.2000.6851

5. Liu, B: $A_{r}^{\lambda}(\Omega)$-weighted imbedding inequalities for A-harmonic tensors. J Math Anal Appl. 273(2), $667-676$ (2002) doi:10.1016/S0022-247X(02)00331-1

6. Liu, B: $A_{r}(\lambda)$-weighted Caccioppoli-type and Poincare-type inequalities for A-harmonic tensors. Int J Math Math Sci. 31 115-122 (2002). doi:10.1155/S0161171202107046

7. Neugebauer, CJ: Inserting Ap-weights. Proc Am Math Soc. 87, 644-648 (1983)

8. Stein, EM: Harmonic Analysis. Princeton University Press, Princeton (1993)

9. Wang, Y, Wu, Y: Sobolev imbedding theorems and Poincare inequalities for Green's operator on solutions of the nonhomogeneous A-harmonic equation. Comput Math Appl. 47, 1545-1554 (2004). doi:10.1016/j.camwa.2004.06.006

10. Xing, Y: Weighted integral inequalities for solutions of the A-harmonic equation. J Math Anal Appl. 279, 350-363 (2003). doi:10.1016/S0022-247X(03)00036-2

11. Xing, Y: Weighted Poincare-type estimates for conjugate A-harmonic tensors. J Inequal Appl. 1, 1-6 (2005)

12. Ding, S, Shi, $P$ : Weighted Poincaré-type inequalities for differential forms in $L^{5}(\mu)$-averaging domains. J Math Anal Appl. 227, $200-215$ (1998). doi:10.1006/jmaa.1998.6096

13. Ding, S: New weighted integral inequalities for differential forms in some domains. Pacific J Math. 149, 43-56 (2000)

14. Ding, S, Nolder, CA: Weighted Poincaré-type inequalities for solutions to the A-harmonic equation. Illinois J Math. 2, 199-205 (2002)

15. Ding, S, Nolder, CA: L $L^{5}(\mu)$-averaging domains. J Math Anal Appl. 283, 85-99 (2003). doi:10.1016/S0022-247X(03)00216-6

16. Nolder, CA: Hardy-Littlewood theorems for A-harmonic tensors. Illinois J Math. 43, 613-631 (1999)

17. Cartan, H: Differential Forms. Houghton Mifflin Co., Boston (1970)

18. Iwaniec, T, Lutoborski, A: Integral estimates for null Lagrangians. Arch Ration Mech Anal. 125, 25-79 (1993). doi:10.1007/ BF00411477

19. Staples, SG: $L^{p}$-averaging domains and the Poincaré inequality. Ann Acad Sci Fenn, Ser Al Math. 14, 103-127 (1989)

20. Staples, SG: Averaging domains: from Euclidean spaces to homogeneous spaces. Proc of Conference on Differential \& Difference Equations and Applications. pp. 1041-1048.Hindawi Publishing (2006)

doi:10.1186/1029-242X-2012-32

Cite this article as: Xing: A new weight class and Poincaré inequalities with the Radon measure. Journal of Inequalities and Applications 2012 2012:32. 